\title{
Firm Growth Function and Extended-Gibrat's Property
}

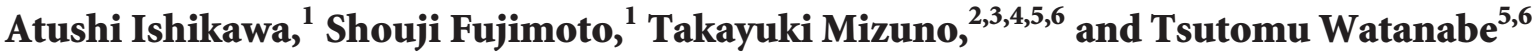 \\ ${ }^{1}$ Kanazawa Gakuin University, 10 Sue, Kanazawa, Ishikawa 920-1392, Japan \\ ${ }^{2}$ National Institute of Informatics, 2-1-2 Hitotsubashi, Chiyoda-ku, Tokyo 101-8430, Japan \\ ${ }^{3}$ Department of Informatics, The Graduate University for Advanced Studies, 2-1-2 Hitotsubashi, Chiyoda-ku, Tokyo 101-8430, Japan \\ ${ }^{4}$ PRESTO, Japan Science and Technology Agency, 7 Gobancho, Chiyodaku, Tokyo 102-0076, Japan \\ ${ }^{5}$ Graduate School of Economics, University of Tokyo, 7-3-1 Hongo, Bunkyo-ku, Tokyo 113-0033, Japan \\ ${ }^{6}$ The Canon Institute for Global Studies, 5-1 Marunouchi 1-chome, Chiyoda-ku, Tokyo 100-6511, Japan \\ Correspondence should be addressed to Atushi Ishikawa; at.ishikawa@gmail.com
}

Received 21 September 2015; Accepted 6 March 2016

Academic Editor: Doojin Ryu

Copyright (C) 2016 Atushi Ishikawa et al. This is an open access article distributed under the Creative Commons Attribution License, which permits unrestricted use, distribution, and reproduction in any medium, provided the original work is properly cited.

\begin{abstract}
We analytically show that the logarithmic average sales of firms first follow power-law growth and subsequently follow exponential growth, if the growth-rate distributions of the sales obey the extended-Gibrat's property and Gibrat's law. Here, the extendedGibrat's property and Gibrat's law are statistically observed in short-term data, which denote the dependence of the growth-rate distributions on the initial values. In the derivation, we analytically show that the parameter of the extended-Gibrat's property is identical to the power-law growth exponent and that it also decides the parameter of the exponential growth. By employing around one million bits of exhaustive sales data of Japanese firms in the ORBIS database, we confirmed our analytic results.
\end{abstract}

\section{Introduction}

As in natural science, statistical laws are also frequently observed in social science. In econophysics, the statistical laws observed in the behavior of people and firms and their universality have been thoroughly investigated $[1,2]$. The statistical laws observed in various social quantities at a point in time are especially well known. One of the main arguments is power-law distribution [3-23]. The power-law distributions of firm-size variables $x_{T}$ in calendar year $T$ (e.g., sales, assets, and number of employees) over size threshold $x_{\text {th }}$ have been observed in a number of years and countries as follows:

$$
P\left(x_{T}\right) \propto x_{T}^{-\mu-1} \text { for } x_{T}>x_{\text {th }} .
$$

Here, $P\left(x_{T}\right)$ is the probability density function (PDF) of $x_{T}$. Exponent $\mu$ is called Pareto's index [3]. At the same time, midsized variables under size threshold $x_{\text {th }}$ have also frequently been described by log-normal distribution.

Short-term statistical laws have also been investigated, which were observed in firm-size variables at two successive points in time $\left(x_{T}, x_{T+1}\right)$ : (quasi-)inverse symmetry and (non-)Gibrat's law. Inverse symmetry denotes that the system is static and symmetric under the time reversal exchange of variables $x_{T} \leftrightarrow x_{T+1}[24,25]$. Quasi-inverse symmetry means that the system is quasi-static and can be expressed as symmetric under the exchange of variables $x_{T} \leftrightarrow a x_{T+1}{ }^{\theta}$, where $a$ and $\theta$ are parameters [26, 27]. Gibrat's law suggests that conditional PDF $Q\left(R \mid x_{T}\right)$ of the growth rate defined by $R=x_{T+1} / x_{T}$ is independent of initial value $x_{T}[24,25]$. This law is observed in the large-scale range over size threshold $x_{\text {th }}$. Non-Gibrat's law, which reflects the dependence of growth-rate distribution $Q\left(R \mid x_{T}\right)$ on initial value $x_{T}$, is observed in the mid-scale range under $x_{\text {th }}$ [28-31].

The statistical laws, which are observed in firm-size variables $x_{T}$ at a point in time, are related to the statistical laws observed in them at two successive points in time $\left(x_{T}, x_{T+1}\right)$. Fujiwara et al. showed that the power law (1) observed in large-sized variables is derived from Gibrat's law $[32,33]$ under inverse symmetry $[24,25]$. The log-normal distribution observed in mid-sized variables is inferred from non-Gibrat's law under inverse symmetry [28-31]. Quasistatistically varying power-law and log-normal distributions are derived from Gibrat's and non-Gibrat's laws under quasiinverse symmetry, respectively $[26,27]$. 
At the same time, interesting studies, related to long-term statistical laws, have been reported in empirical data analyses of the network of firms. Miura et al. showed that the number of business connections of firms exponentially increases as they age [34]. Mizuno et al. reported that the sales of firms depend on the number of business connections [35]. Such observations suggest that the sales of firms exponentially grow with age. In this context, the emergence of an early power-law growth is natural for firms that started small. Small firms must grow rapidly to reach the trajectory of exponential growth. This result is probably related to Luttmer's report that the number of employees $L$ grows rapidly in the beginning but soon loses momentum [36]. This can be interpreted as power-law growth. Besides firm-size variables, Petersena et al. observed power-law growth in the progress of researchers defined by the number of publications of scientific papers [37].

In this study, we propose a law of the newly formulated firm growth derived from short-term statistical laws. If there are laws in firm growth [38], they are long-term statistical laws that are different from statistical laws in the shortterm. However, these laws might be related. We verify this conjecture in the rest of our paper, which is organized as follows. The law of firm growth must be related to nonGibrat's and Gibrat's laws that describe the change of the growth-rate distributions that are dependent on the initial values. In the next section, we review Gibrat's law and introduce an extended-Gibrat's property instead of nonGibrat's law. We also analytically derive firm growth that first obeys the power-law function and subsequently follows an exponential function. After that, using our database, we demonstrate that firm growth is analytically derived and observed in the empirical data. The last section concludes this paper.

\section{Results}

2.1. Gibrat's Law and Extended-Gibrat's Property. Gibrat's law is represented using conditional $\operatorname{PDF} Q\left(R \mid x_{T}\right)$ as follows:

$$
Q\left(R \mid x_{T}\right)=Q(R) \quad \text { for } x_{T}>x_{\mathrm{th}} .
$$

Here, $Q\left(R \mid x_{T}\right)$ does not depend on initial value $x_{T}$. This Gibrat's law is observed in a range over size threshold $x_{\text {th }}$. On the other hand, under threshold $x_{\mathrm{th}}, Q\left(R \mid x_{T}\right)$ does depend on $x_{T}$. For the case of sales [31], the positive growthrate distributions gradually decrease as $x_{T}$ increases, and the negative growth-rate distributions hardly change. Using conditional PDF $q\left(r \mid x_{T}\right)$ of logarithmic growth rate $r=$ $\log _{10} R$ which is related to $Q\left(R \mid x_{T}\right)$ by $q\left(r \mid x_{T}\right)=$ $\ln 10 R Q\left(R \mid x_{T}\right)$, this property can be expressed as

$$
\begin{aligned}
& q\left(r \mid x_{T}\right)=\frac{f(r)}{x_{T}^{1 / \gamma}} \quad \text { for } r>0, \\
& q\left(r \mid x_{T}\right)=g(r) \quad \text { for } r<0,
\end{aligned}
$$

where $\gamma$ is a constant. In this paper, we call (3) and (4) the extended-Gibrat's property, which is different from the previously examined non-Gibrat's law [30, 31]. Later, we confirm the extended-Gibrat's property with empirical data.
2.2. Analytical Derivation of Firm Growth. Next, we analytically derive firm growth from the extended-Gibrat's property ((3) and (4)) and from Gibrat's law (2). To estimate firm growth, it is convenient to identify a starting point in time for every firm. We consider a firm's incorporation as its starting point and consider its age from its year of foundation. For simplicity, we examine the average values instead of the distribution of variables and denote the logarithmic average value of the firm-size variable at firm age $t$ as $x_{t}$. The foundation year is $t=1$.

We define the growth rate from $x_{t}$ to $x_{t+1}$ as

$$
R\left(x_{t}\right)=\frac{x_{t+1}}{x_{t}}
$$

The dependence in growth rate $R$ on $x_{t}$ is significant in the extended-Gibrat's property. We hypothesize that Gibrat's law and the extended-Gibrat's property, which are observed in two successive calendar years $(T, T+1)$, are also valid in two successive firm ages $(t, t+1)$. Therefore, in the following discussions, calendar year $T$ in $Q\left(R \mid x_{T}\right)$ can be replaced by firm age $t$.

In extended-Gibrat's property range $x_{t}<x_{\text {th }}$, using (3) and (4), the average value of $r$ is expressed as

$$
\begin{aligned}
r\left(x_{t}\right) & =\int_{-\infty}^{\infty} d r r q\left(r \mid x_{t}\right) \\
& =\frac{1}{x_{t}^{1 / \gamma}} \int_{0}^{\infty} d r r f(r)+\int_{-\infty}^{0} d r r g(r) \\
& =\frac{A}{x_{t}^{1 / \gamma}}+B,
\end{aligned}
$$

where integrations $\int_{0}^{\infty} d r r f(r)$ and $\int_{-\infty}^{0} d r r g(r)$ are assumed to converge and are denoted as $A$ and $B$, respectively. In (6), the dependence of $r\left(x_{t}\right)$ on $x_{t}$ is important. When the second term is negligible compared with the first term in (6), $r\left(x_{t}\right)$ is approximated by

$$
\log _{10} r\left(x_{t}\right)=-\frac{1}{\gamma} \log _{10} x_{t}+\log _{10} \gamma+\frac{1}{\gamma} \log _{10} D .
$$

Here, $\log _{10} A=\log _{10} \gamma+(1 / \gamma) \log _{10} D$. Equation (7) can be rewritten as follows:

$$
r\left(x_{t}\right)=\gamma\left(\frac{D}{x_{t}}\right)^{1 / \gamma} .
$$

By combining (5) and (8), we obtain the following recurrence formula:

$$
x_{t+1}=x_{t} 10^{\gamma\left(D / x_{t}\right)^{1 / \gamma}} .
$$

When $t$ is sufficiently larger than $1,(9)$ has a solution:

$$
x_{t}=(\ln 10)^{\gamma} D t^{\gamma} .
$$

We used Maclaurin expansion as $\ln (1+1 / t) \sim 1 / t$. 


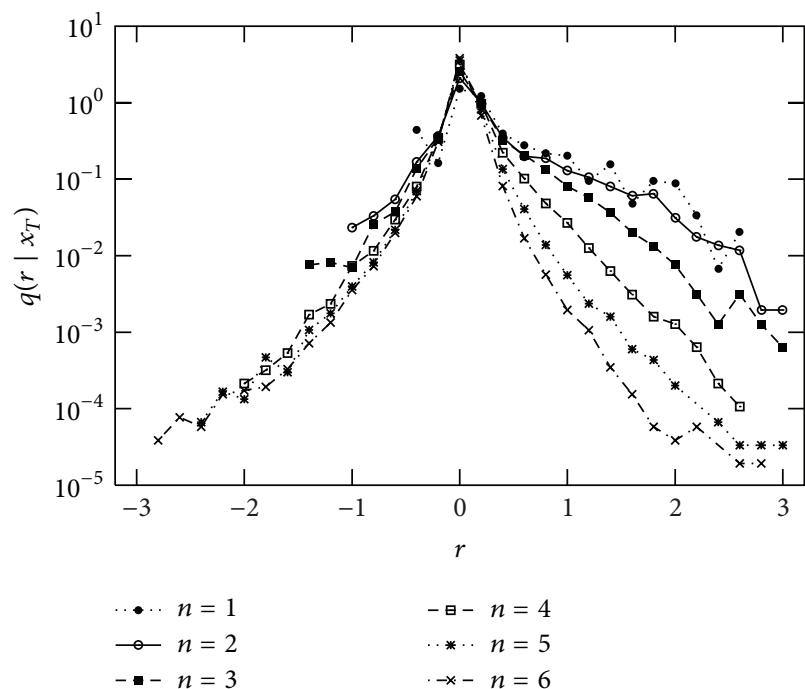

(a)

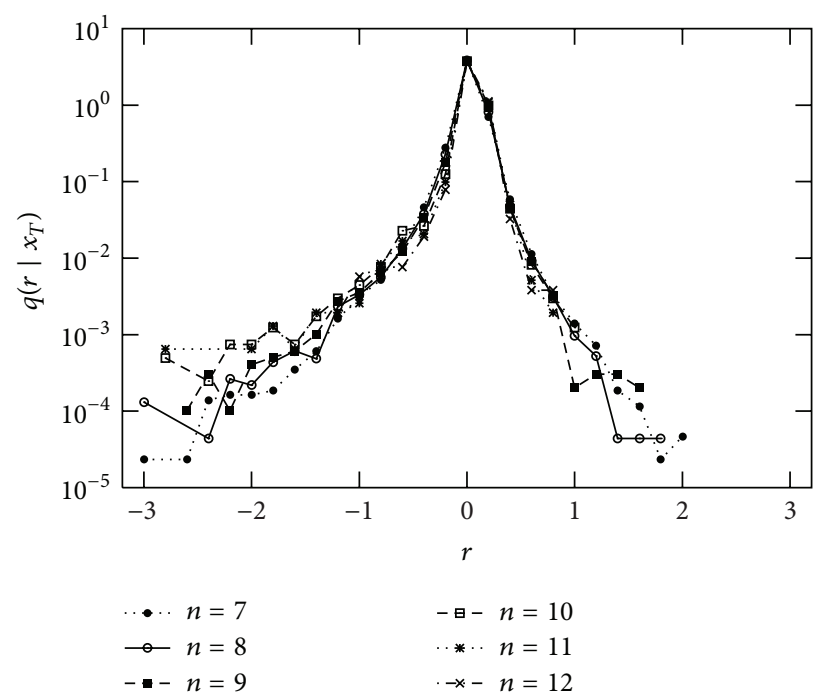

(b)

Figure 1: Conditional PDFs $q\left(r \mid x_{T}\right)$ of logarithmical sales growth rate $r=\log _{10} x_{T+1} / x_{T}$ in logarithmically equal-sized bins $x_{T} \in$ $\left[10^{0.5(n-1)}, 10^{0.5 n}\right]((\mathrm{a}) n=1,2, \ldots, 6$; (b) $n=7,8, \ldots, 12)$ of Japanese firms in ORBIS database. Here, $x_{T}$ and $x_{T+1}$ are sales in consecutive years $T=2007$ in thousands of US dollars.

In Gibrat's law range $x_{t}>x_{\text {th }}$, the average value of $r$ is a constant: $\gamma\left(D / x_{\mathrm{th}}\right)^{1 / \gamma}$. Therefore, the recurrence formula takes the following form:

$$
x_{t+1}=x_{t} C,
$$

where $C=10^{\gamma\left(D / x_{\mathrm{th}}\right)^{1 / \gamma}}$ is a constant. Equation (11) has a solution:

$$
x_{t}=C^{\prime} \exp [\beta t] .
$$

In (12), $C^{\prime}$ is a constant and $\beta$ is given by

$$
\beta=\gamma\left(\frac{D}{x_{\text {th }}}\right)^{1 / \gamma} \ln 10 .
$$

Consequently, using the extended-Gibrat's property and Gibrat's law, we show that the growth of the logarithmic average of sales $x_{t}$ first follows a power-law function when $t \gg 1$ under $x_{\text {th }}$ and next follows an exponential function above $x_{\text {th }}$. In this discussion, the parameter of the extendedGibrat's property $\gamma$ is identified by the power-law exponent, and $\gamma$ also decides the parameter of exponential growth $\beta$.

2.3. Data Analysis. We employ the ORBIS database, provided by Bureau van Dijk [39], which contains around 150 million pieces of firm-size data from all over the world. In the database, we analyze around one million pieces of Japanese firm-size data for 2007 and 2008. Since the number of active firms in Japan is estimated to be around one million [40], this database is exhaustive. We denote the sales data of Japanese firms from 2007 and 2008 as $\left(x_{T}, x_{T+1}\right)$ with $T=2007$.

First, we confirm Gibrat's law (2) and the extendedGibrat's property $((3)$ and (4)). Figure 1 depicts the conditional PDFs $q\left(r \mid x_{T}\right)$ of logarithmical sales growth $r$ in

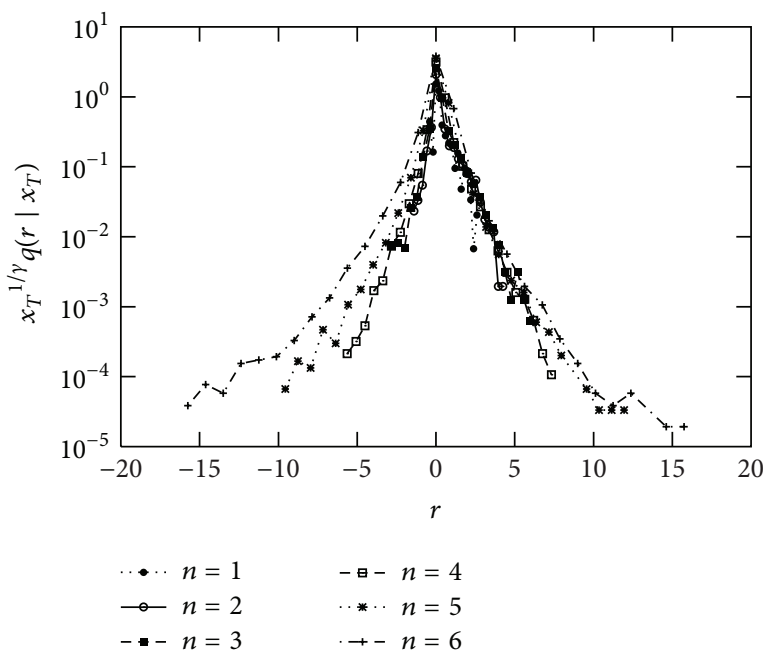

FIgURE 2: Conditional PDFs $q\left(r \mid x_{T}\right)$ times $x_{T}{ }^{1 / \gamma}$ with $\gamma=3.3$ in Figure 1(a).

logarithmically equal-sized bins $x_{T} \in\left[10^{0.5(n-1)}, 10^{0.5 n}\right](n=$ $1,2, \ldots, 12)$ in thousands of US dollars. The values $\left(x_{T}, x_{T+1}\right)$ are adjusted using the Consumer Price Index. In Figure 1(b), where $x_{T}>10^{3}$, the growth-rate distributions hardly change as $n$ increases. This corresponds to Gibrat's law (2). On the other hand, in Figure 1(a) where $x_{T}<10^{3}$, the positive growth-rate distributions gradually decrease as $n$ increases, and the negative growth-rate distributions hardly change. The later property corresponds to (4). Figure 2 shows that, for $r>0, x_{T}^{1 / \gamma} q\left(r \mid x_{T}\right)$ hardly changes as $n$ increases when $\gamma=3.3$. This figure also verifies (3). 


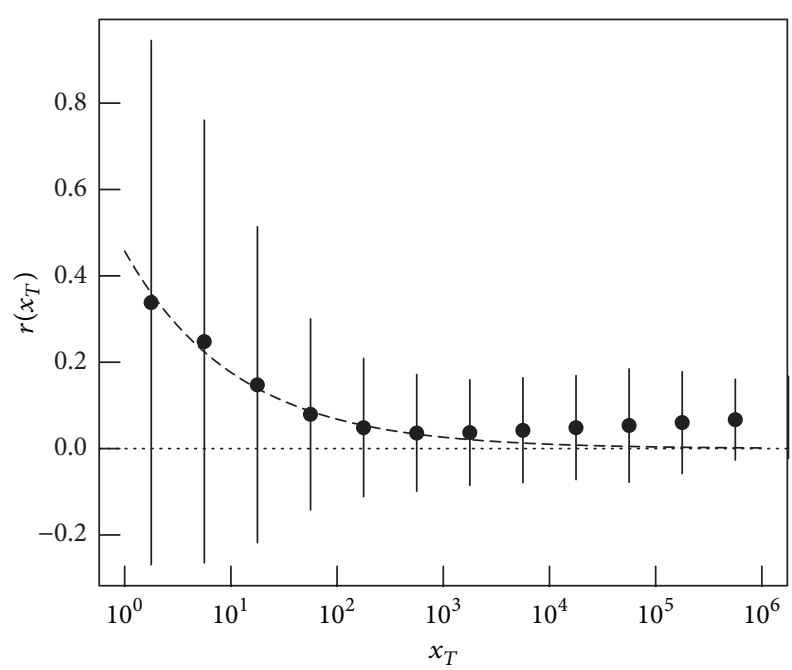

FIGURE 3: Scatterplot between logarithmical intermediate value $x_{T}=10^{0.5(n-1 / 2)}$ of each bin $\left[10^{0.5(n-1)}, 10^{0.5 n}\right](n=1,2, \ldots, 12)$ in thousands of US dollars and logarithmic average value $r\left(x_{T}\right)$ of $r$ in each bin in Figure 1. Standard deviations are also depicted as error bars. Dashed line is an optimal exponential curve for $x_{T}<10^{3}$, and dotted line is $r\left(x_{T}\right)=0$.

Figure 3 shows logarithmic mean value $r\left(x_{T}\right)$ and the standard derivation in each bin $\left[10^{0.5(n-1)}, 10^{0.5 n}\right](n=$ $1,2, \ldots, 12)$ in Figure 1. In Figure $3, x_{T}$ is the logarithmical intermediate value of each bin, where $x_{T}=10^{0.5(n-1 / 2)}$. Average values $r\left(x_{T}\right)$ exponentially decrease as the logarithm of $x_{T}$ increases in the range under $10^{3}$. This corresponds to the extended-Gibrat's property in the mid-scale range of sales data less than $x_{\text {th }} \sim 10^{3}$, and (7) is confirmed. On the other hand, average values $r\left(x_{T}\right)$ hardly change in the large-scale range over $10^{3}$. This observation verifies Gibrat's law (2) over a threshold value whose size is $x_{\text {th }} \sim 10^{3}$.

Second, to verify the analytical derivation of firm growth, we compared the parameters estimated in different ways using empirical data. From Figures 1 and 3, we used $x_{\text {th }} \sim 10^{3}$. Applying (7) to the data points in Figure 3 under $x_{\text {th }} \sim 10^{3}$, $\gamma$ and $D$ are estimated to be $2.42 \pm 0.13$ and $0.018 \pm 0.010$, respectively. From these values, (13) gives $\beta=0.061 \pm 0.031$. On the other hand, in Figure 2, $\gamma$ is set to 3.3. In Gibrat's law range $x_{t}>x_{\text {th }}$ of Figure 3, constant average $r$ can be estimated. From this value, $\beta$ is directly calculated to be $0.11 \pm 0.01$. Parameters $\beta$ and $\gamma$ estimated in one way are very close to the values differently calculated in another way. Using short-term data, we checked the consistency of the analytical derivation of firm growth.

Note that since this derivation is based on average values, we must consider distribution. Figure 3 also provides information about the distribution of the values. In a Gibrat's law range where $x_{t}>x_{\text {th }} \sim 10^{3}$, the standard deviation of logarithmic average values $r\left(x_{t}\right)$ hardly changes. However, in the extended-Gibrat's property range where $x_{t}<x_{\text {th }} \sim 10^{3}$, the standard deviation becomes larger as $x_{t}$ becomes smaller. This indicates that exponent $\gamma$ of the early power-law growth deviates widely until the exponential growth begins, whereas parameter $\beta$ of the exponential growth does not deviate as much as $\gamma$.

In the analytical derivation of firm growth, we assumed that the shapes of Figures 1, 2, and 3 resemble other years that are not $T=2007$ and that Gibrat's law and the extendedGibrat's property are valid in two successive firm ages $(t, t+1)$. Confirming this assumption in the examined ORBIS database is difficult, because it does not include long-term data. For the same reason, the growth of firms cannot be observed directly by tracing the history of the sales of each firm. However, the database contains not only firm-size data but also the incorporation years of firms. Therefore, the growth of the average value of sales $x_{t}((10)$ and (12)) that are analytically derived can be approximately observed under the above assumption.

Third, using these data, we can observe the firm growth derived in the previous subsection. In Figure 4(a), by classifying the sales of Japanese firms in 2007 that are included in the database into age-rank bins with a 1-year width, the logarithmic averages of sales $x_{t}$ in each bin $(t=1,2, \ldots, 50)$ are depicted by circles in a single logarithmic plot. Similarly, the logarithmical averages of sales in 2008 in Japan are also depicted by crosses. In this figure, to adjust the growth in 2007 to the growth in 2008, sales $x_{t}$ in 2007 are divided by the total amount of sales in 2007 and multiplied by the total sales in 2008. In Figure 4(b), the logarithmic averages are plotted in a $\log$-log scale. In Figure 4, the dashed line is an optimal powerlaw function, and the dotted line is an optimal exponential function. These figures show that, under $x_{\text {th }} \sim 10^{3}, x_{t}$ is well fitted by the power-law function (10). At the same time, over $x_{\text {th }} \sim 10^{3}, x_{t}$ is well fitted by an exponential function (12). Using the least square method, the parameters of exponential growth $\beta$ and power-law growth $\gamma$ are estimated by $\beta=$ $0.022 \pm 0.006$ and $\gamma=0.89 \pm 0.05$, respectively. These parameters that are estimated by long-term data are not close to the parameters of the short-term data. This discrepancy probably comes from the breakdown of the assumption that the growth rate of sales with respect to firm age was always distributed in a similar shape between 2007 and 2008. At the same time, in Figure 4, the power-law growth (10) is not smoothly continuous to the exponential growth (12) because of the distortion of sales from ages 15 to 20. This corresponds to Japan's bubble economy from 1987 to 1992.

\section{Discussion}

In this study, we analytically showed that the logarithmic average sales of firms first follow a power-law growth and subsequently an exponential growth, if the growth-rate distributions of the sales obey the extended-Gibrat's property and Gibrat's law. Here, the extended-Gibrat's property and Gibrat's law are statistically observed in the short-term data for two successive years, which denote the dependence of the growth-rate distributions on the initial values. By employing the exhaustive sales data of Japanese firms in 2007 and 2008 that are contained in the ORBIS database, first, we confirmed the extended-Gibrat's property and Gibrat's law. 


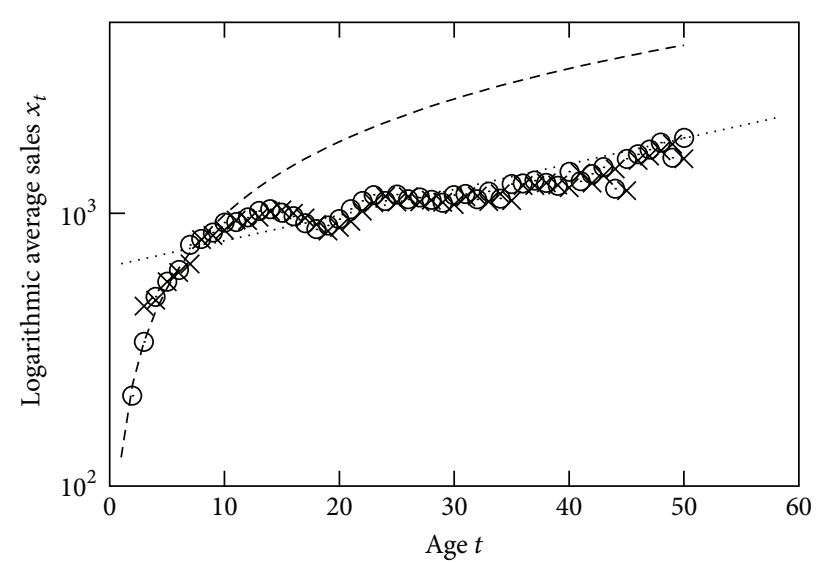

(a)

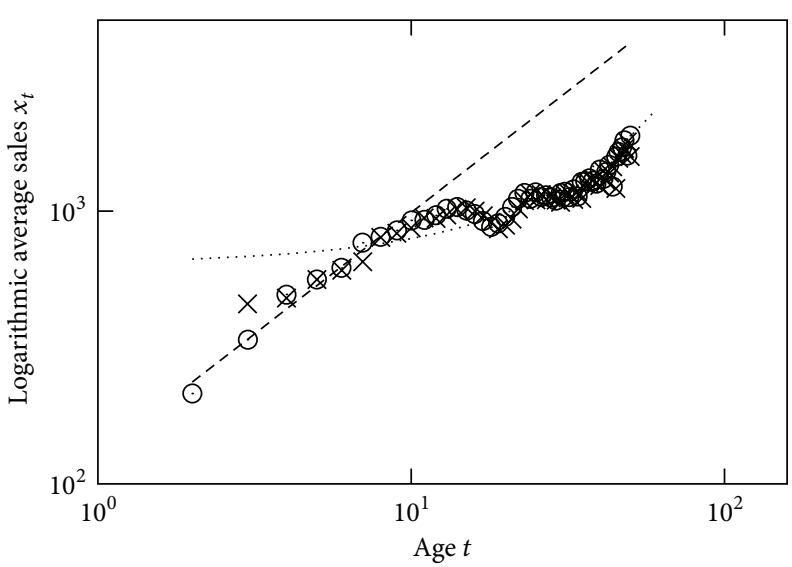

(b)

FIGURE 4: (a) By classifying sales of firms in 2007 in Japan included in ORBIS database into age-rank bins with 1-year width, logarithmic average of sales in each bin $(t=1,2, \ldots, 50)$ is depicted by circles in single logarithmic plots. Similarly, logarithmic average of sales in 2008 in Japan is also depicted by crosses. (b) Logarithmic average of sales $x_{t}$ classified into age-rank bins with a 1-year width is plotted in double logarithmic plots. In both figures, dashed and dotted lines represent optimal power-law and optimal exponential functions, respectively.

At the same time, we checked the consistency of the analytic derivation of firm growth by comparing the parameters of the extended-Gibrat's property, Gibrat's law, the power-law growth, and the exponential growth in short-term data. After that, we investigated the average firm growth of sales in 2007 and 2008 from Japan with respect to firm ages using the firm incorporation years in the database. We observed early power-law growth and subsequent exponential growth.

In the analytic derivation of firm growth, we did not assume inverse symmetry [24, 25] or non-Gibrat's law [2831]. Instead, we proposed an extended-Gibrat's property under size threshold $x_{\text {th }}$, which is important to power-law growth. Gibrat's law over $x_{\text {th }}$ is also important to exponential growth. Similar growths are known as the $S$-curve of a product. In $S$-curves, expansion periods correspond to power-law growth and later maturity periods correspond to exponential growth. The average growth rate of sales definitely exceeds 1 even in a Gibrat's law range where $x_{t}>x_{\text {th }}$. This feature is different from the growth of a product, which follows an $S$ curve.

It is fascinating that the extended-Gibrat's property and Gibrat's law, which are observed in short-term statistical data, can explain both power-law and exponential growth, which are long-term statistical laws. The boundary between Gibrat's and the extended-Gibrat's ranges presented in this study $x_{\text {th }}$ is determined in all kinds of industries and firms in Japan. Perhaps it differs with respect to kinds of industries and other factors. In this case, mega firms might exist, which grow under a power law beyond average boundary $x_{\text {th }}$.

The discussions in this paper, constructed by analyzing sales data, are applicable to other firm-size variables, such as assets, number of employees, and positive profits. The results obtained here can also be applied to GDP growth analyses. The growth-rate distributions of GDP, which follow the extended-Gibrat's property, decide a GDP's power-law growth and exponential growth. This is not only an intriguing theme but also a significant one for such advanced countries that are suffering from sluggish GDP growth as Japan and the United States.

\section{Competing Interests}

The authors declare that they have no competing interests.

\section{Acknowledgments}

This study was supported by JSPS KAKENHI Grant nos. 24510212, 24710156.

\section{References}

[1] R. N. Mantegna and H. E. Stanley, Introduction to Econophysics: Correlations and Complexity in Finance, Clarendon Press, Oxford, UK, 1991.

[2] A. Saichev, Y. Malevergne, and D. Sornette, Theory of Zip's Law and Beyond, Cambridge University Press, Cambridge, UK, 2009.

[3] V. Pareto, Cours d'Economie Politique, Macmillan, London, UK, 1897.

[4] M. E. J. Newman, "Power laws, Pareto distributions and Zipf's law," Contemporary Physics, vol. 46, no. 5, pp. 323-351, 2005.

[5] A. Clauset, C. R. Shalizi, and M. E. J. Newman, "Power-law distributions in empirical data," SIAM Review, vol. 51, no. 4, pp. 661-703, 2009.

[6] E. Bonabeau and L. Dagorn, "Possible universality in the size distribution of fish schools," Physical Review E, vol. 51, no. 6, pp. R5220-R5223, 1995.

[7] S. Redner, "How popular is your paper? An empirical study of the citation distribution," European Physical Journal B, vol. 4, no. 2, pp. 131-134, 1998.

[8] Y. Fujiwara and H. Aoyama, "Large-scale structure of a nationwide production network," European Physical Journal B, vol. 77, no. 4, pp. 565-580, 2010. 
[9] H. Aoyama, W. Souma, Y. Nagahara, M. P. Okazaki, H. Takayasu, and M. Takayasu, "Pareto's law for income of individuals and debt of bankrupt companies," Fractals, vol. 8, no. 3, pp. 293-300, 2000.

[10] T. Yamano, "Distribution of the Japanese posted land price and the generalized entropy," European Physical Journal B, vol. 38, no. 4, pp. 665-669, 2004.

[11] R. N. Mantegna and H. E. Stanley, "Scaling Behaviour in the dynamics of an economic index," Nature, vol. 376, no. 6535, pp. 46-49, 1995.

[12] R. L. Axtell, “Zipf distribution of U.S. firm sizes," Science, vol. 293, no. 5536, pp. 1818-1820, 2001.

[13] B. Podobnik, D. Horvatic, A. M. Petersen, B. Urošević, and H. E. Stanley, "Bankruptcy risk model and empirical tests," Proceedings of the National Academy of Sciences of the United States of America, vol. 107, no. 43, pp. 18325-18330, 2010.

[14] D. Fu, F. Pammolli, S. V. Buldyrev et al., "The growth of business firms: theoretical framework and empirical evidence," Proceedings of the National Academy of Sciences of the United States of America, vol. 102, no. 52, pp. 18801-18806, 2005.

[15] B. Podobnik, D. Horvatic, F. Pammolli, F. Wang, H. E. Stanley, and I. Grosse, "Size-dependent standard deviation for growth rates: empirical results and theoretical modeling," Physical Review E, vol. 77, no. 5, Article ID 056102, 2008.

[16] K. Okuyama, M. Takayasu, and H. Takayasu, "Zipf's law in income distribution of companies," Physica A, vol. 269, no. 1, pp. 125-131, 1999.

[17] P. Gopikrishnan, M. Meyer, L. A. N. Amaral, and H. E. Stanley, "Inverse cubic law for the distribution of stock price variations," European Physical Journal B, vol. 3, no. 2, pp. 139-140, 1998.

[18] T. Mizuno, M. Katori, H. Takayasu, and M. Takayasu, "Statistical laws in the income of Japanese companies," in Empirical Science of Financial Fluctuations, pp. 321-330, Springer, Tokyo, Japan, 2002.

[19] M. Nirei and W. Souma, "A two factor model of income distribution dynamics," Review of Income and Wealth, vol. 53, no. 3, pp. 440-459, 2007.

[20] H. Iyetomi, H. Aoyama, Y. Fujiwara, Y. Ikeda, and W. Souma, "A paradigm shift from production function to production copula: statistical description of production activity of firms," Quantitative Finance, vol. 12, no. 9, pp. 1453-1466, 2012.

[21] M. Levy and S. Solomon, "Power laws are logarithmic Boltzmann laws," International Journal of Modern Physics C, vol. 7, no. 4, pp. 595-601, 1996.

[22] D. Sornette and R. Cont, "Convergent multiplicative processes repelled from zero: power laws and truncated power laws," Journal de Physique I, vol. 7, pp. 431-444, 1997.

[23] H. Takayasu, A.-H. Sato, and M. Takayasu, "Stable infinite variance fluctuations in randomly amplified langevin systems," Physical Review Letters, vol. 79, no. 6, pp. 966-969, 1997.

[24] Y. Fujiwara, W. Souma, H. Aoyama, T. Kaizoji, and M. Aoki, "Growth and fluctuations of personal income," Physica A, vol. 321, no. 3-4, pp. 598-604, 2003.

[25] Y. Fujiwara, C. Di Guilmi, H. Aoyama, M. Gallegati, and W. Souma, "Do Pareto-Zipf and Gibrat laws hold true? An analysis with European firms," Physica A, vol. 335, no. 1-2, pp. 197-216, 2004.

[26] A. Ishikawa, "Annual change of Pareto index dynamically deduced from the law of detailed quasi-balance," Physica A: Statistical Mechanics and its Applications, vol. 371, no. 2, pp. 525535, 2006.
[27] A. Ishikawa, "Quasi-statically varying power-law and lognormal distributions in the large and the middle scale regions of japanese land prices," Progress of Theoretical Physics Supplement, vol. 179, pp. 103-113, 2009.

[28] A. Ishikawa, "Derivation of the distribution from extended Gibrat's law," Physica A, vol. 367, pp. 425-434, 2006.

[29] A. Ishikawa, "The uniqueness of firm size distribution function from tent-shaped growth rate distribution," Physica A: Statistical Mechanics and its Applications, vol. 383, no. 1, pp. 79-84, 2007.

[30] M. Tomoyose, S. Fujimoto, and A. Ishikawa, "Non-Gibrat's law in the middle scale region," Progress of Theoretical Physics Supplements, vol. 179, pp. 114-122, 2009.

[31] A. Ishikawa, S. Fujimoto, and T. Mizuno, "Shape of growth rate distribution determines the type of Non-Gibrat's property," Physica A, vol. 390, no. 23-24, pp. 4273-4285, 2011.

[32] R. Gibra, Les Inégalités Économique, Sirey, Paris, France, 1932.

[33] J. Sutton, "Gibrat's legacy," Journal of Economic Literature, vol. 35, no. 1, pp. 40-59, 1997.

[34] W. Miura, H. Takayasu, and M. Takayasu, "Effect of coagulation of nodes in an evolving complex network," Physical Review Letters, vol. 108, no. 16, Article ID 168701, 2012.

[35] T. Mizuno, W. Souma, and T. Watanabe, "The structure and evolution of buyer-supplier networks," PLoS ONE, vol. 9, no. 7, Article ID e100712, 2014.

[36] E. G. J. Luttmer, "On the mechanics of firm growth," Review of Economic Studies, vol. 78, no. 3, pp. 1042-1068, 2011.

[37] A. M. Petersena, M. Riccaboni, H. E. Stanley, and F. Pammolli, "Persistence and uncertainty in the academic career," Proceedings of the National Academy of Sciences of the United States of America, vol. 109, no. 14, pp. 5213-5218, 2012.

[38] A. Coad, The Growth of Firms, Edward Elgar, 2009.

[39] Bureau van Dijk, http://www.bvdinfo.com/Home.aspx/.

[40] Statistics Bureau, "Ministry of Internal Affairs and Communications," http://www.stat.go.jp/english/index.htm. 


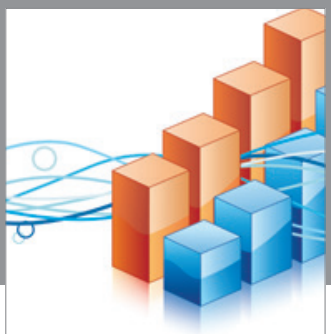

Advances in

Operations Research

vatem alat4

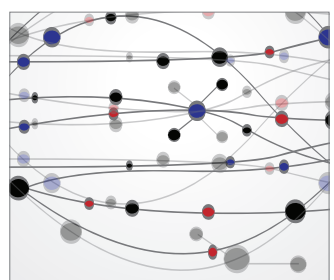

\section{The Scientific} World Journal
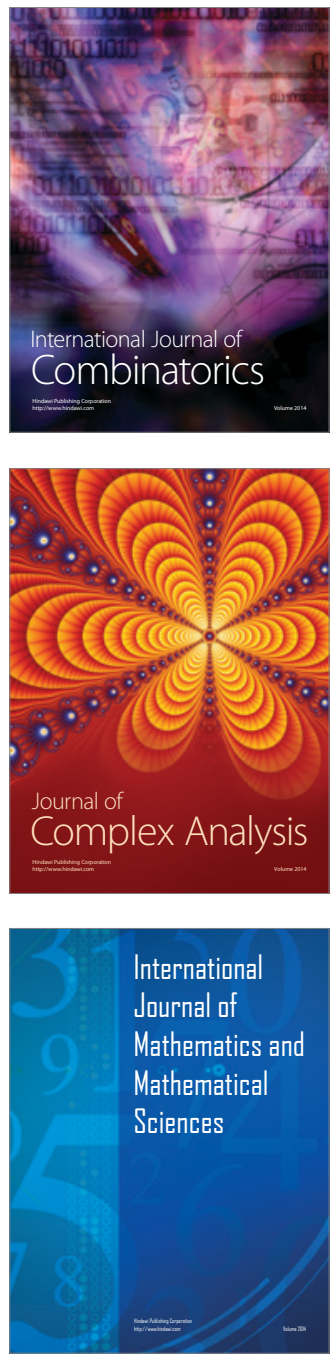
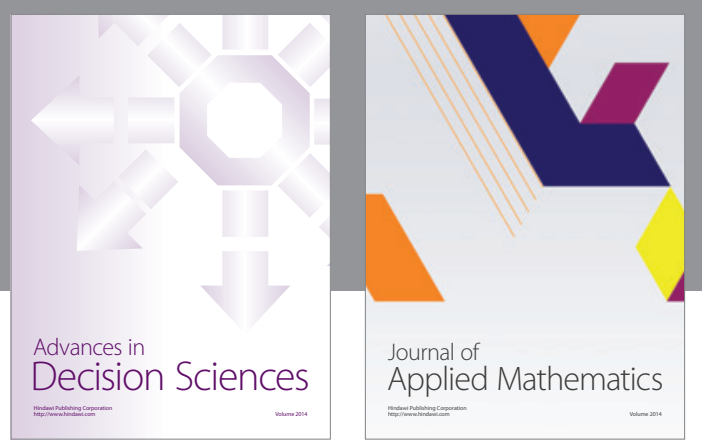

Algebra

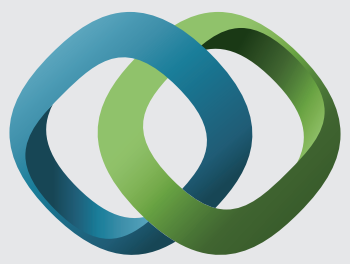

\section{Hindawi}

Submit your manuscripts at

http://www.hindawi.com
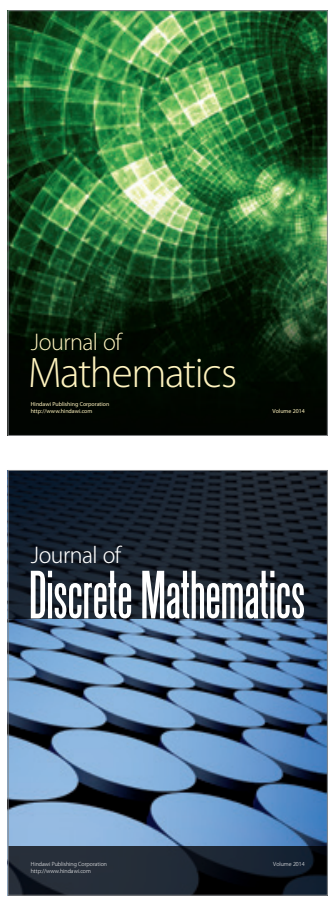

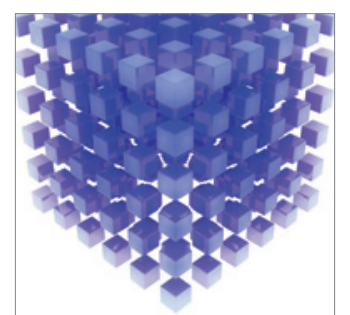

Mathematical Problems in Engineering
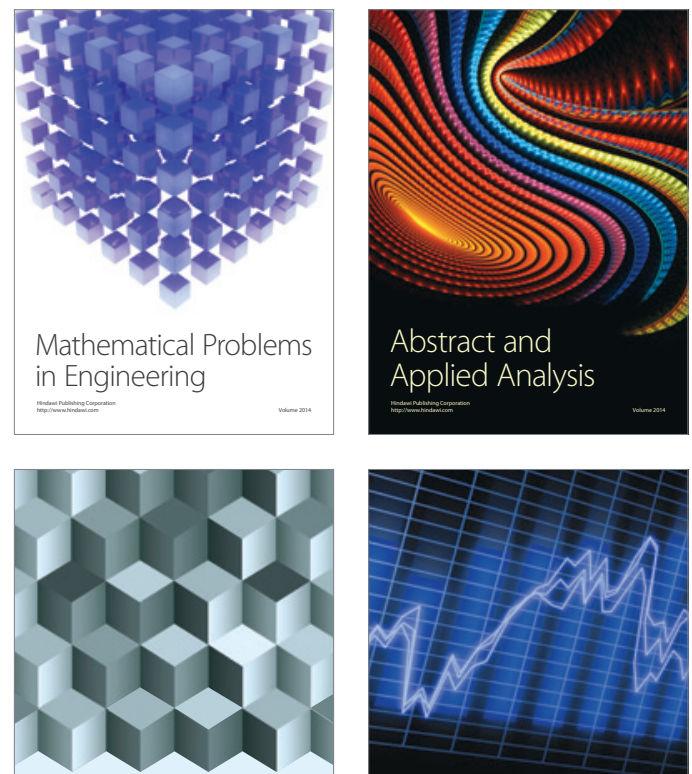

Journal of

Function Spaces

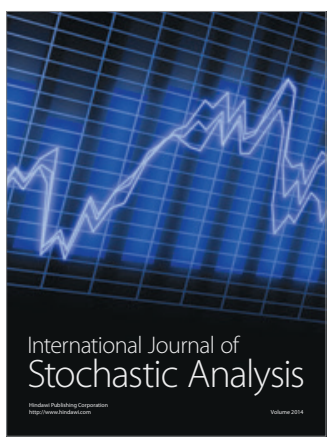

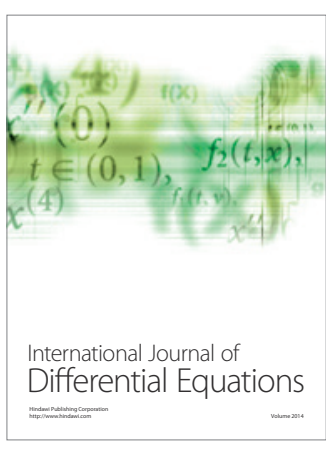
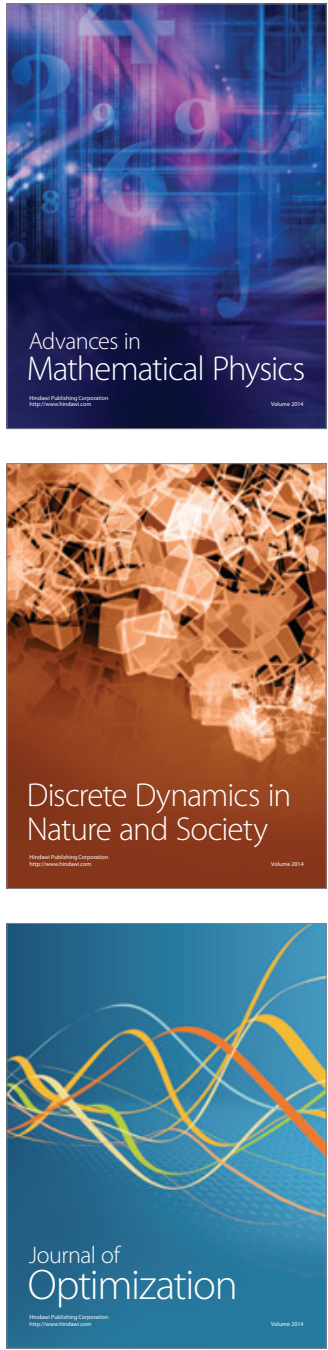